\title{
Assembly 4.0 - Flexibly Picked Up, Precisely Mounted
}

\author{
Matthias Bock and Markus Kleineberg German Aerospace Center DLR
}

Citation: Bock, M. and Kleineberg, M., "Assembly 4.0 - Flexibly Picked Up, Precisely Mounted," SAE Technical Paper 2019-01-1355, 2019, doi:10.4271/2019-01-1355.

\section{Abstract}

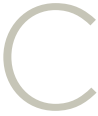

omponent deviations between as-designed and as-built as a result of deformations in the production process lead to significant cost increasing during assembly. After quality inspection, components are provisionally preassembled to determine their matching accuracy. Components with an acceptable tolerance range are then assembled. Otherwise costly reworks are required. Subsequently, the preassembly process is repeated until acceptable assembly tolerances are achieved. To avoid this time-consuming and costly process loop, a flexible mounting frame coupled with a real-time measuring system is used to optimize the assembly process. The flexible mounting frame can be applied for many applications where components are to be aligned and mounted. This flexible mounting frame allows the realization of exact shape and acceptable position tolerances.

The mounting frame consists of telescopic carbon fiber reinforced polymer (CFRP) tubes, linear actuators and suctions grippers. Linear actuators deform the component in the elastic range. Connected vacuum suction grippers hold the component securely to the mounting frame. The individually configurable joining device compensates manufacturing deviations during assembly in the elastic range. This is realized through a real time 3-D surface measuring system that assists in controlling a variable number of actuators. The geometric deviations are detected contactless by an external measuring system. This system provides the required measurements to control the actuators. By decoupling the measuring method from the manipulator, the system design can be kept very simple without compromising accuracy.

Furthermore, the frame is mounted on a mobile robot platform. The applied mobile robot platform consists of the mobile platform (KUKA omniMove) and an industrial robot (KR QUANTEC 150 R3300 prime K). By using such mobile robot, the system can be universally used. Moreover, components can be brought into the assembly position by contour optimized elastic deformation. This novel implementation of flexibly pick up and precisely mounting system results in a more efficient and faster assembly.

\section{Introduction}

$\mathrm{n}$ the aerospace industry, structural components of CFRP (Carbon Fiber Reinforced Plastic) are used with rising trend. Furthermore, the application of composite materials is planned for mass production [1]. Therefore the lightweight construction aspect is a dominant factor in aeronautical structures and thus influential in the design of the subcomponents and their assembly. While metal components in addition to the elastic deformability also provide plastic deformation, thermoset fiber composite components only provide an elastic deformability.

Form and position deviations due to internal stresses are usually visible only on the finished component. Even qualityassured mass production of CFRP components can not completely prevent contour deviations, especially with complex-shaped or stiffened components. Unfortunately even small deviations cause in the post-processing and final assembly of such components a large time and thus economic effort.

After quality inspection, components are provisionally preassembled to determine their matching accuracy. Components with an acceptable tolerance range are then assembled. Otherwise costly reworks are required.
Subsequently, the preassembly process is repeated until acceptable assembly tolerances are achieved (compare Figure 1).

The standard measures for geometry correction is the "shimming", in which gaps are filled up with fillers. Since the "shimming" is performed essentially manually and is very time-consuming due to the required curing process, significant cost and time risks arise outside the direct value added.

\section{FIGURE 1 Assembly process for an aircraft wing made of} fiber-reinforced plastics.

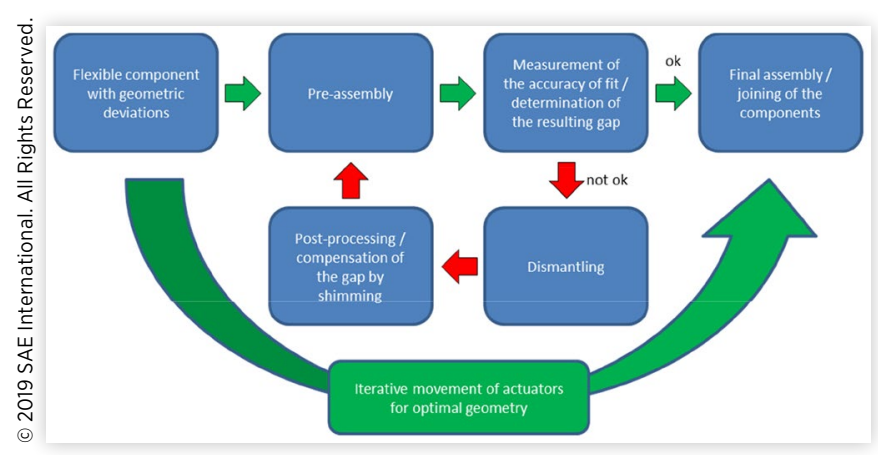




\section{Mounting Frame to Remove Deviations from the Production Process with Smart Assembly}

In order to avoid time-consuming and costly process loops during assembly, a form-flexible mounting frame is developed and verified, which uses the elastic deformation potential of a subcomponent for the assembly process.

The approach is based on introducing a subcomponent as close as possible to the nominal dimension geometry by introducing bending moments and then assembling it.

The geometric deviations are detected in real time by an external contactless measurement device which makes data available for the control of the actuators. Due to the consistent decoupling of measuring technology and actuator technology, the manipulator equipped with electric linear actuators can be designed very simply and without great demands on rigidity.

\section{CFRP Tubes}

The mounting frame consists of telescopic CFRP tubes. The high-precision tubes were manufactured in the prepreg winding process. The core layers are with unidirectional fiber orientation, the cover layers of plain weave fabric. Three tubes with an external diameter of $60 \mathrm{~mm}$ and a wall thickness of $5 \mathrm{~mm}$ serve as horizontal crossbeams. They are $2000 \mathrm{~mm}$ long and can optionally be extended to $4000 \mathrm{~mm}$. CFRP tubes are also used as tube connectors for telescoping the horizontal crossbeams. These pipe pieces are $1000 \mathrm{~mm}$ long and have an outer diameter of $50 \mathrm{~mm}$ with the same wall thickness. On sectional view these tubes are arranged as a triangle.

At these crossbeams, telescopic tubes are arranged as a framework. The outer tubes have an inner diameter of $25 \mathrm{~mm}$, the inner tubes have an inner diameter of $24 \mathrm{~mm}$ with a wall thickness of $0.5 \mathrm{~mm}$. They can be extended from a length of $1000 \mathrm{~mm}$ to $1800 \mathrm{~mm}$. By clamping with clamping rings, the tubes are fixed at the appropriate length (see Figure 2).

FIGURE 2 Telescopic CFRP tubes with clamping rings.

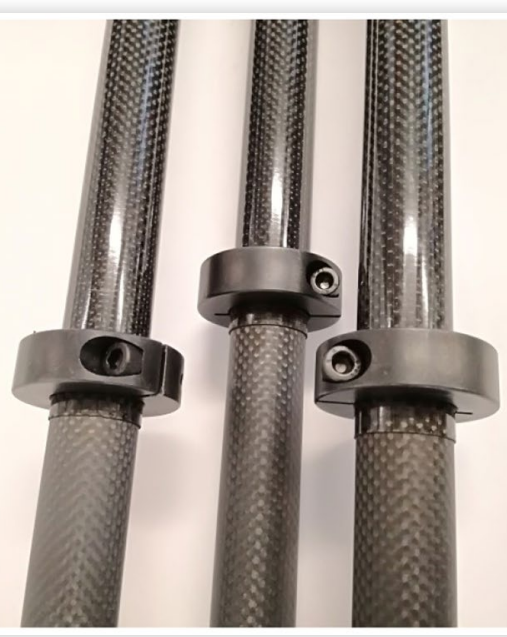

\section{FIGURE 3 2-piece sleeve element with built-in CFRP tube.}

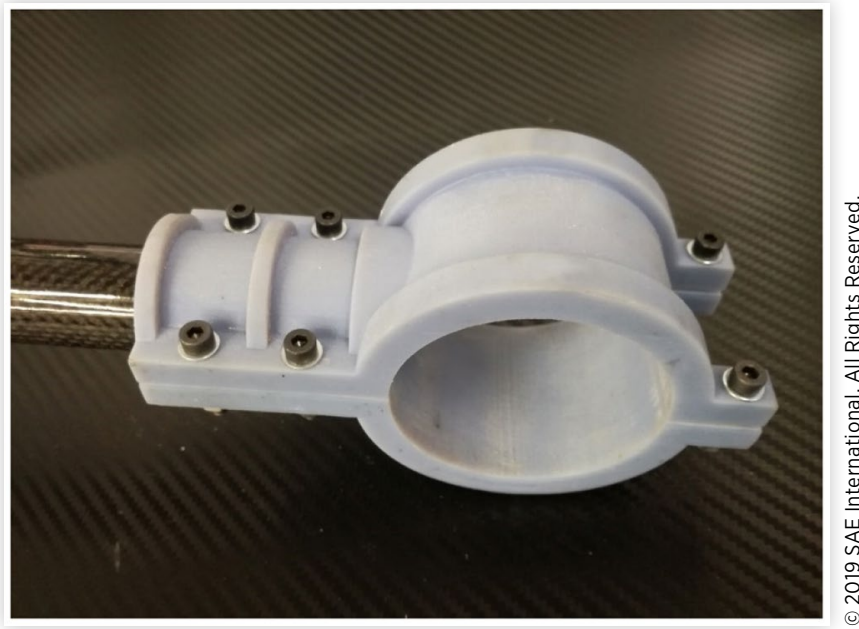

They are mounted to the crossbeams with 3-D printed two-piece T-sleeve elements (see Figure 3).

Linear actuators to deform the component in the elastic range are connected to the CFK pipes via adapter plates.

\section{Component Manipulator Interface}

The linear actuators are NEMA 17 linear actuators. This 2-phase hybrid stepper motor has a maximum force of $200 \mathrm{~N}$. The maximum stroke is $25.4 \mathrm{~mm}$. In order to have maximum adjustment possibilities, the actuators are positioned at the beginning of the measurement in the middle position. The maximum usable stroke is thus $\pm 12.7 \mathrm{~mm}$.

The stepper motors are controlled by the Kocomotion stepper motor controller MForce Motion Control. Each actuator has its own controller in multi-axis operation via an RS485 bus [2]. The system is connected to the measuring system via a USB coupler.

For maximum flexibility to accommodate different geometries, 9 telescopic lengths are used. These are variable in length as well as in angle. For this purpose, the telescopic fixings are unlocked and placed manually on the component with the vacuum suction grippers. Then the vacuum suction grippers are switched on individually (see Figure 4). These connected vacuum suction grippers hold the component securely on the mounting position.

Thereafter, the telescopic tubes are locked in length and the component can be moved freely.

\section{3-D Measurement Method (ATOS)}

Nowadays, non-contact measuring systems are rising in engineering metrology due to their efficiency. In particular, when measuring parts could not be measured with coordinate measuring machines or could be measured very complicated [3]. As a non-contact measuring system, the ATOS Triple Scan System from GOM is used. Here, a precise stripe pattern is 
FIGURE 4 Linear actuators with vacuum suction grippers to fix the component.

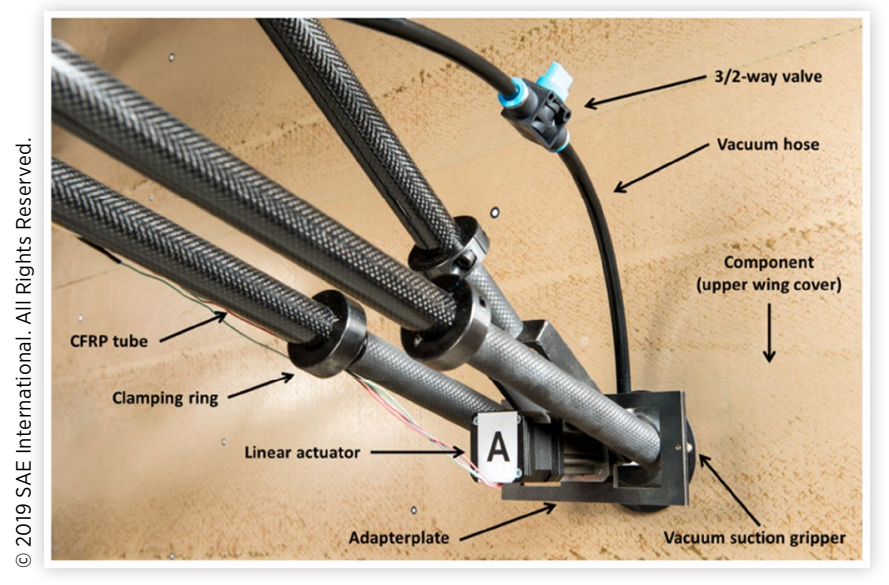

FIGURE 5 ATOS measurement of the upper wing cover.

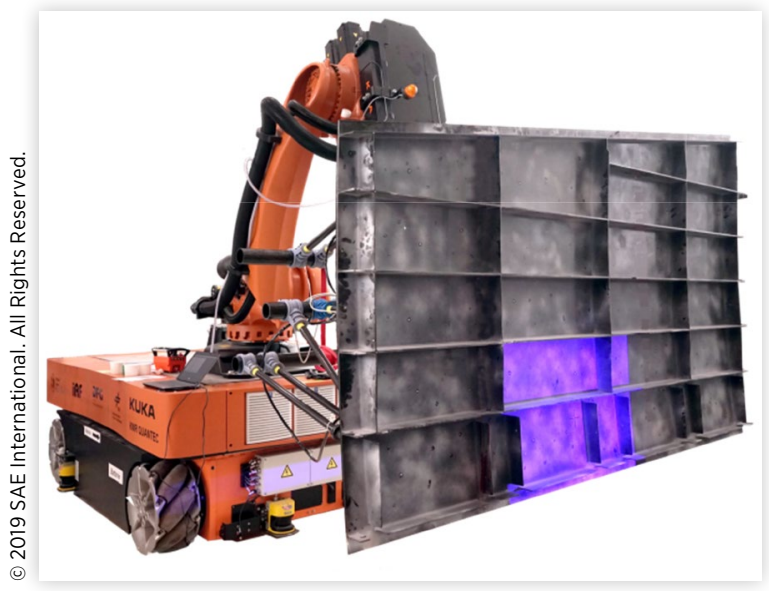

projected onto the object surface and recorded by two cameras using the stereo camera principle [4]. The ATOS optical 3-D scanner is based on the principle of triangulation using a stereo camera setup. This stereo camera setup and a projection unit are integrated in the ATOS sensor head. The sensor projects different fringe patterns onto the scanned objects surface (see Figure 5). These patterns are recorded by the two CCD cameras (left and right), forming a phase shift based upon sinusoidal intensity distributions on the CCD chips. The ATOS uses multiple phase shifts in a heterodyne principle to achieve highest sub-pixel accuracy. Based on the optical transformation equations, independent 3D coordinates are automatically calculated for each camera pixel []].

\section{Mobile Mounting Frame}

The flexible mounting frame can be used wherever large and complex components (such as CFRP, GRP, plastic, metal, etc.) need to be aligned and mounted.

After picking up and deforming the component in the elastic range, it is transported and installed in nominal geometry for assembly. For this purpose, the mounting frame

\section{FIGURE 6 Flexible mounting frame with mobile robot} platform.

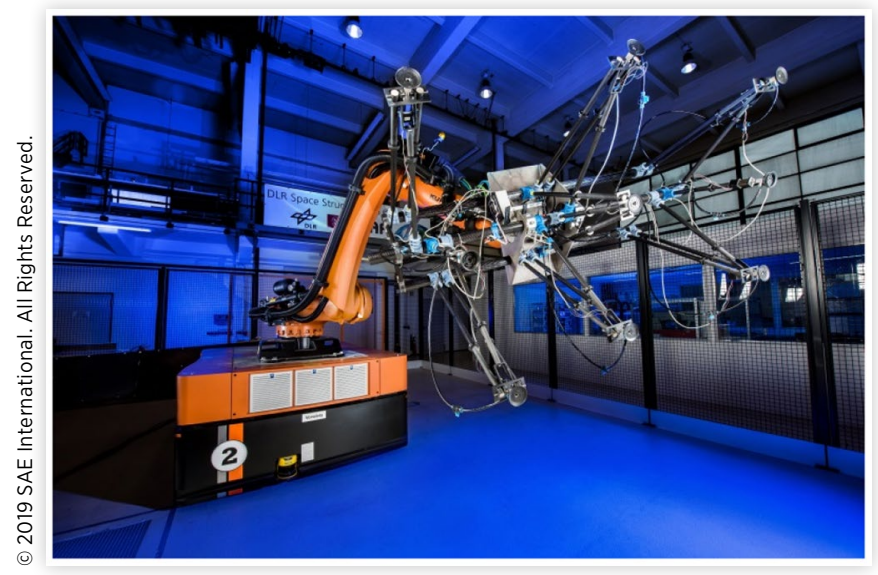

is installed on a mobile robot platform (Kuka KMR Quantec) (Figure 6). This can significantly simplify the assembly of fiber composite components.

The KMR QUANTEC consists of the mobile platform KUKA omniMove and a KUKA robot of the KR QUANTEC series. The basic idea behind the KMR Quantec is to overcome the limitations in the reach of classic robots with a mobile platform. This allows the robots to demonstrate significantly more flexible production processes than would be possible with gantry systems or rail systems. A stationary plant needs a permanent place in the production hall. With mobile robots, production areas can be used as needed and make it possible to produce very large components. This makes the mobile platforms particularly suitable for the production of large components. In addition to the production of CFRP components, the robot will also be used for other production processes such as the assembly of large structures [6]. By taking over transport tasks, the mobile platform offers great advantages here.

Each wheel can move independently of the others. This means the mobile platform can also move sideways, diagonally and even in a circle. So it moves safely and accurately in a confined space. Thus, the mobile robot unit offers maximum flexibility and almost unlimited mobility.

The integrated robot is a KR QUANTEC 150 R3300 prime K. According to data sheet, it has a load capacity of 150 kilograms with a range of 3,300 millimeters [7].

High-performance batteries ensure a self-sufficient power supply for the platform, as well as for the robot on the platform and its periphery.

\section{Validation of the System Demonstrated on the Assembly of an Upper Wing Cover}

The validation of the system was carried out in the Clean Sky 2 project NACOR (New Innovative Aircraft Designations and 


\section{FIGURE 6 Wing cover assembly with assembly rig.}

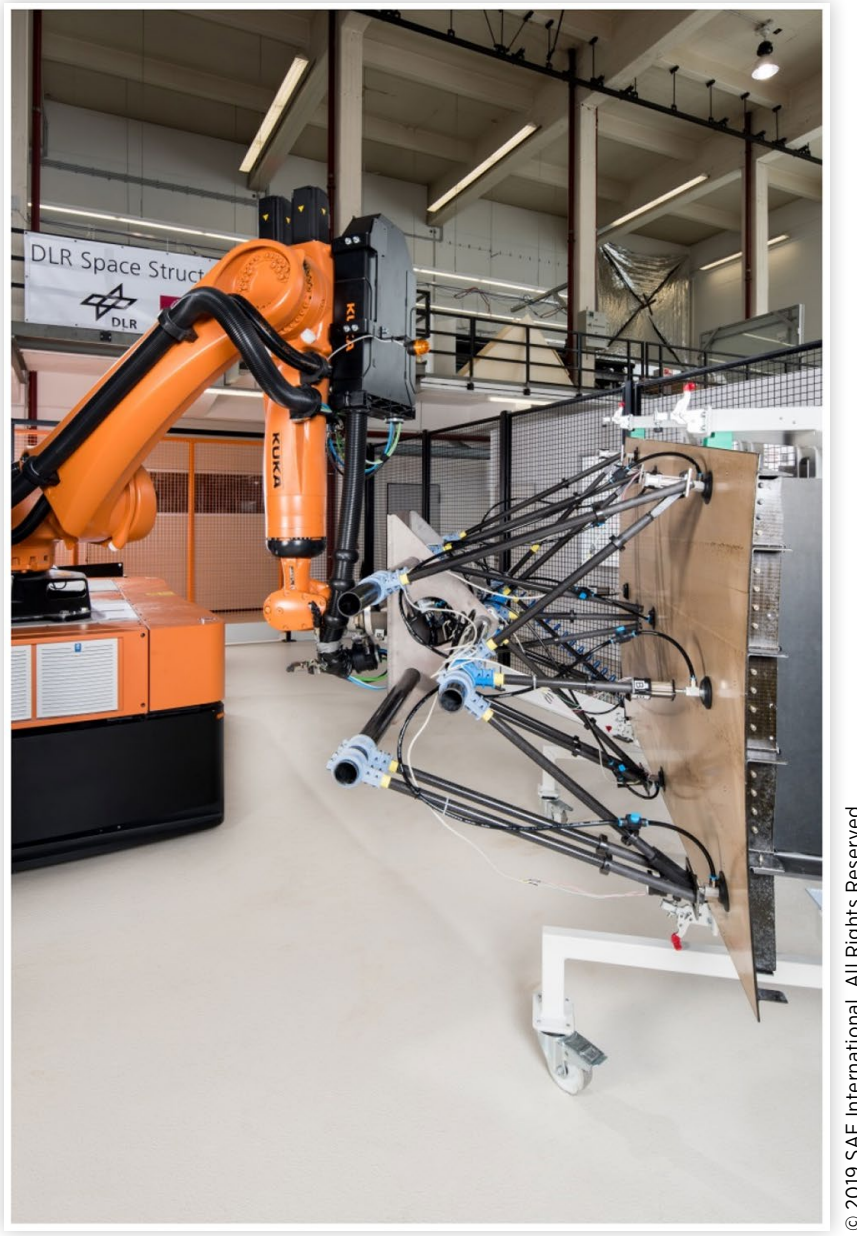

Related Issues), which investigates the possibilities of laminar airfoil support.

The Ground Based Demonstrator that has to be mounted, consists of an integral upper wing cover, a flat front spar and 5 wing ribs with integrated L-angle. In the project, the mobile mounting frame was used to connect the laminar compatible wing upper cover with ribs and front spar.

For the assembly with specific deformation, the upper wing cover is assembled to the mounting frame. For this purpose, the wing cover is placed with laminar surface upwards in front of the Omnimove. The mounting frame is positioned $4 \mathrm{~cm}$ above the component in the middle. Subsequently, the individual vacuum suction grippers are placed on specific positions and turned on. Thereafter, the clamping rings are tightened to fix the tubes. After all nine grippers were fixed and turned on, the wing cover is placed vertically (see Figure 7). Afterwards, the elastic deformation of the wing cover begins.

The first step is to measure the cover with the sensor system as shown in Figure 7. Based on this data, which has been compared with the CAD, the strategy for the actuation is selected. Subsequently, the actuators are adjusted by the corresponding compensation value. Initially, the saddle shape is minimized before pushing over the horizontal centerline to align the edges. The actuators are moved individually by a certain length after each measurement.
FIGURE 7 First measurement after picking up the wing cover without adjusting the actuators.

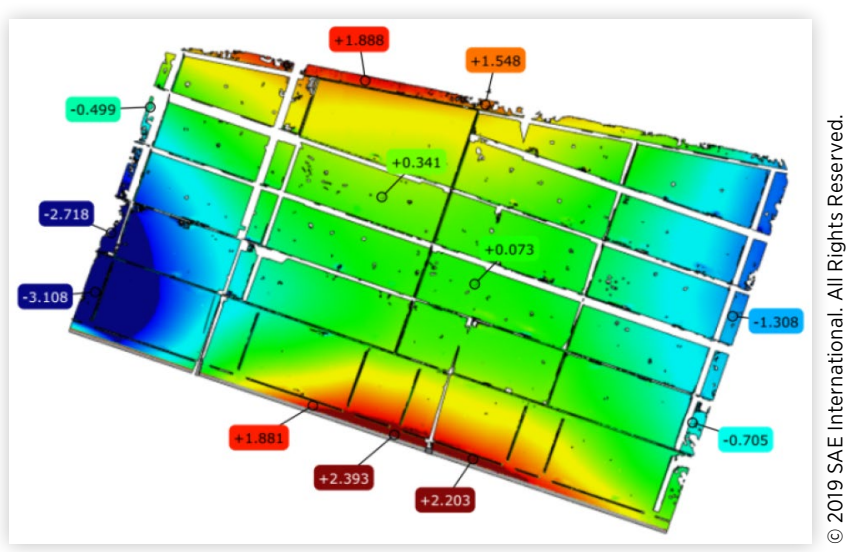

FIGURE 8 Result after adjustment step 4.

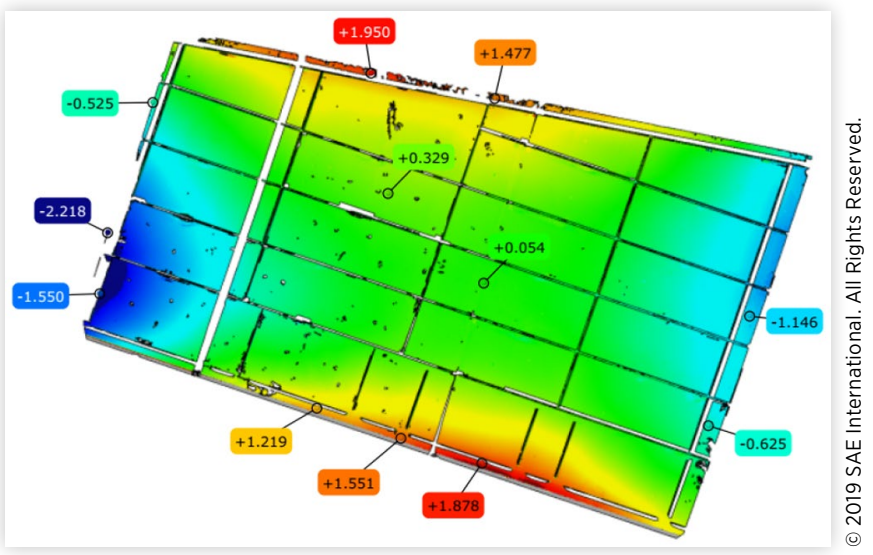

\section{FIGURE 9 Final result after adjustment step 9.}

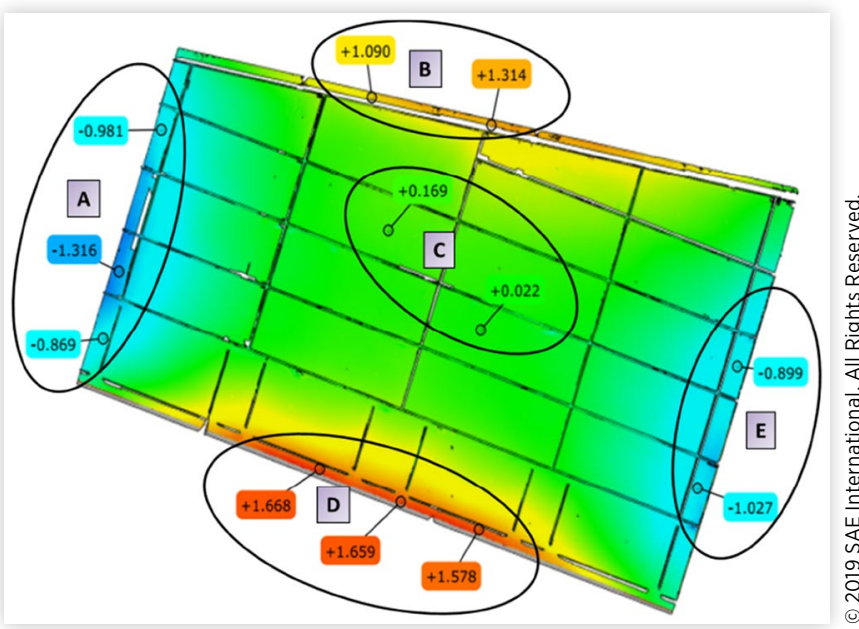

Using the principle of contactless measurement and actuation, the elasticity of the upper wing cover was successfully used to reduce the mean deviation by $32.5 \%$ after nine adjustment steps. For a closer consideration, the measurements were divided into five groups. This clearly shows the increase in accuracy in a more global context (see Table 1). 
TABLE 1 Improvement of the deviations.

\begin{tabular}{|l|lll|} 
Group & $\begin{array}{l}\text { Sum of deviations - } \\
\text { at the beginning }\end{array}$ & $\begin{array}{l}\text { Sum of deviations - } \\
\text { at the end }\end{array}$ & Improvement \\
\hline A & $6,325 \mathrm{~mm}$ & $3,166 \mathrm{~mm}$ & $49,9 \%$ \\
\hline B & $3,436 \mathrm{~mm}$ & $2,404 \mathrm{~mm}$ & $30,0 \%$ \\
\hline C & $0,414 \mathrm{~mm}$ & $0,191 \mathrm{~mm}$ & $53,9 \%$ \\
\hline D & $6,476 \mathrm{~mm}$ & $4,905 \mathrm{~mm}$ & $24,3 \%$ \\
\hline E & $2,013 \mathrm{~mm}$ & $1,926 \mathrm{~mm}$ & $4,3 \%$ \\
\hline
\end{tabular}

\section{Outlook}

The next stage of the mobile mounting frame, the acquisition of the assembly data should be focused. In particular, the deformation forces in conjunction with the associated structural deformation can be used as an assessment approach for the quality and reproducibility of the process. Hereby, the existing gap of the "digital twin" in the field of assembly can be significantly reduced. Currently, suitable sensors are being studied that fit well into the overall concept. In addition, it will be investigated whether a special variation of the geometry during the joining process brings advantages. For example, the part could be put on in a kind of rolling motion. Thereby shim masses or adhesives can be controlled and reproducibly distributed in the gap and reduced in quantity.

\section{Summary/Conclusions}

Deviations in the production process can lead to enormous costs during assembly.

In order to avoid time-consuming and costly process loops during assembly, a form-flexible mounting frame is developed and verified in which the previously unused elastic deformation potential of a subcomponent for the assembly process is used.

The approach is based on initiating a subcomponent as close as possible to the nominal geometry by introducing bending moments before the component is assembled.

For this purpose, a mounting frame made of telescopic CFRP tubes, to accommodate and deform components with large geometric variation. These tubes are equipped with vacuum suction grippers mounted on linear actuators.

The geometric deviations are detected in real time by an external contactless device and made available for the control of the actuators. These deform the component in the elastic range in target geometry. By using a mobile robot, the component can be transported and directly assembled in the mounting position.
The approach of non-contact measurement and actuation by a mounting frame was successfully used to exploit the elasticity of an upper wing cover and thus to reduce the mean deviation from the ideal geometry by $32.5 \%$.

\section{References}

1. Krombholz, C., Perner, M., Bock, M., and Röstermundt, D., "Improving the Production Quality of the Advanced Automated Fiber Placement Process by Means of Online Path Correction," in 28th Congress of the International Council of the Aeronautical Sciences, 2012, 1-10.

2. Kocomotion, "MForce Motion Control Stepper Datasheet," n.d..

3. Vagovský, J., Buranský, I., and Görög, A., "Evaluation of Measuring Capability of the Optical 3D Scanner," Procedia Engineering 100(Jan.):1198-1206, 2015, doi:10.1016/j. proeng.2015.01.484.

4. GOM GmbH, "ATOS Triple Scan,” n.d., https://www.gom. com/de/messsysteme/atos/atos-triple-scan.html.

5. Morovic, L. and Pokorny, P., "Optical 3D Scanning of Small Parts," Automation Equipment and Systems, Pts 1-4 468-471: 2269-2273, 2012, doi:10.4028/www.scientific.net/AMR.468$\underline{471.2269}$.

6. Neue Braunschweiger Zeitung, "Weltweit Einmalig," Multimedia Werbe- Und Verlags-GmbH, Jan. 9, 2016.

7. KUKA, “Specification KR QUANTEC K Prime Robot,” 2018.

\section{Contact Information}

\author{
Dipl.-Ing. Matthias Bock \\ German Aerospace Center \\ Institute of Composite Structures and Adaptive Systems \\ Department Composite Technology \\ Matthias.Bock@dlr.de
}

\section{Acknowledgments}

I would like to thank the Institute of Adaptronics and Function Integration of the Technical University of Braunschweig for their support. Furthermore I would like to thank the company of GOM for the support and the professional exchange.

\section{Definitions/Abbreviations}

CFRP - carbon fiber reinforced polymer

Prepreg - preimpregnated fibers

(c) 2019 SAE International. All rights reserved. No part of this publication may be reproduced, stored in a retrieval system, or transmitted, in any form or by any means, electronic, mechanical, photocopying, recording, or otherwise, without the prior written permission of SAE International. 